\title{
Los azulejos de A Rocha Branca (Padrón, A Coruña): Un estudio arqueométrico
}

\author{
The tiles from A Rocha Branca (Padrón, A Coruña): An archaeometric study \\ http://dx.doi.org/10.15304/gall.37.5411
}

\author{
M. Pilar Prieto Martínez \\ Universidade de Santiago de Compostela \\ pilar.prieto@usc.es
}

Francisco Alonso Toucido
Universidade de Santiago de Compostela
franalonsotoucido@hotmail.com

Óscar Lantes-Suárez

Universidade de Santiago de Compostela

oscar.lantes@usc.es

Fernando Acuña Castroviejo (†)

Universidade de Santiago de Compostela

\section{Raquel Casal García}

Universidade de Santiago de Compostela

\section{Resumen}

Gracias al estudio arqueométrico de azulejos y otros elementos constructivos de lujo recuperados en el castillo medieval de A Rocha Branca (Padrón, Coruña), se ofrecen nuevos datos sobre las técnicas de fabricación de los mismos así como posibles lugares de producción que permiten profundizar en el estudio del comercio de época bajomedieval en Galicia. Se ha procedido a analizar un total de seis fragmentos, cinco de los cuales son azulejos vidriados, bien dibujados o bien monocromos y una pieza también vidriada, interpretada como un marco de puerta o ventana. Se trata de materiales asociados al arzobispo don Lope de Mendoza (1400-1445).

Palabras clave: Azulejos, Rocha Branca, Lope de Mendoza, Arqueometría medieval, Cerámica de lujo, castillo, mitra compostelana.

\begin{abstract}
Thanks to an archaeometric study of the tiles and other luxury construction elements recovered from the mediaeval castle of A Rocha Branca (Padrón, Coruña), new data is provided regarding the manufacturing techniques that were used for them, as well as the locations where they were possibly produced, making it possible to explore the topic of trade during the late medieval period in Galicia in greater detail. A total of six fragments were analysed, five of which are from glazed tiles, which are well-decorated or otherwise monochrome, and another glazed piece, which has been interpreted as part of a door or window frame. These are materials associated with Archbishop Lope de Mendoza (14001445).
\end{abstract}

Keywords: Tiles, Rocha Branca, Lope de Mendoza, mediaeval archaeometry, luxury ceramics, castle, archbishops of Compostela. 


\section{INTRODUCCIÓN}

A pesar de que los estudios de cerámica medieval y postmedieval están empezando a desarrollarse en Galicia, el material constructivo todavía no ha recibido demasiada atención. En el caso del castillo de A Rocha Branca, podemos decir que una parte de estos materiales, los azulejos encargados por el arzobispo don Lope de Mendoza (1400-1445), han llamado la atención y han sido referenciados en varias publicaciones (p. ej. CASAMAR, 1979; CARAMÉS et al., 2006; FERNÁNDEZ ABELLA, 2015). En este trabajo se presentan los resultados preliminares del estudio arqueológico y arqueométrico de 6 piezas representativas seleccionadas de este yacimiento. Es la primera vez que se realizan análisis físico-químicos en material constructivo tipo azulejo en Galicia.

A Rocha Branca (parroquia de Santa María de Iria Flavia, Padrón, A Coruña) es un castillo arzobispal compostelano de época bajomedieval, uno de los tres grandes núcleos fortificados de los señores eclesiásticos de la tierra de Santiago de Compostela. Se trata de un castillo conformado por dos recintos y posee una 'vida y uso del siglo XIII al XV, con una ocupación residual hasta mediados del s. XVII' (FERNÁNDEZ ABELLA, 2015: 417).

La riqueza y singularidad del castillo de Rocha Branca era bien conocida en época medieval, de ello deja buena constancia uno de los documentos más esclarecedores sobre los castillos y torres bajomedievales gallegos, el pleito Tabera-Fonseca ${ }^{1}$. Ya que a Rocha Branca, también sería derruido por los Irmandiños. Son varios los testigos del pleito, que describen en mayor o menor medida la fortaleza, llegándose incluso a mencionar los azulejos como por ejemplo "dicha Rocha Blanca... ...la mas hermosa e deleitosa que abía en todo el Reino... ... abía muchos hedifiçios... ...ricos y fermosos dorados y pintados e ladrillados todo el suelo de azulejos y con sus huertas, naranjales, bosque, fuentes y estanques y otras cosas para deleite...” (YZQUIERDO PERRÍN, 2006-2007: 126).

En el año 1981 se realiza una intervención arqueológica en el castillo, bajo la dirección de los profesores de historia medieval Ermelindo Portela Silva y María del Carmen Pallares Méndez así como del profesor de arqueología Fernando Acuña Castroviejo de la Universidad de Santiago de Compostela. La intervención cubrió un área de 325 metros cuadrados en el recinto conocido como A Barronca. En la misma se constató la cimentación del edificio principal del castillo, una gran torre central, su muro perimetral y su foso. Lamentablemente son escasos los datos de contexto, procedentes de esta intervención (FERNÁNDEZ ABELLA, 2015). Han llegado hasta nosotros algunos materiales constructivos, procedentes de la mencionada intervención, los cuales estudiamos en este trabajo.

1 Pleito iniciado en 1525 (OLIVERA, 2000: 23), entre el recientemente designado arzobispo de Santiago, Juán Tabera y el saliente, Alonso III de Fonseca. Por el estado en el que se encontraba la red de fortificaciones de la mitra compostelana. La mayoría de estas fortalezas se encontraban destruidas, debido principalmente a la revuelta Irmandiña y a la falta de arreglo y mantenimiento posteriores. El arzobispo entrante reclamaba al saliente una indemnización, por ese estado de ruina. Siendo el pleito un referente para conocer la Galicia bajomedieval y sus fortalezas. 


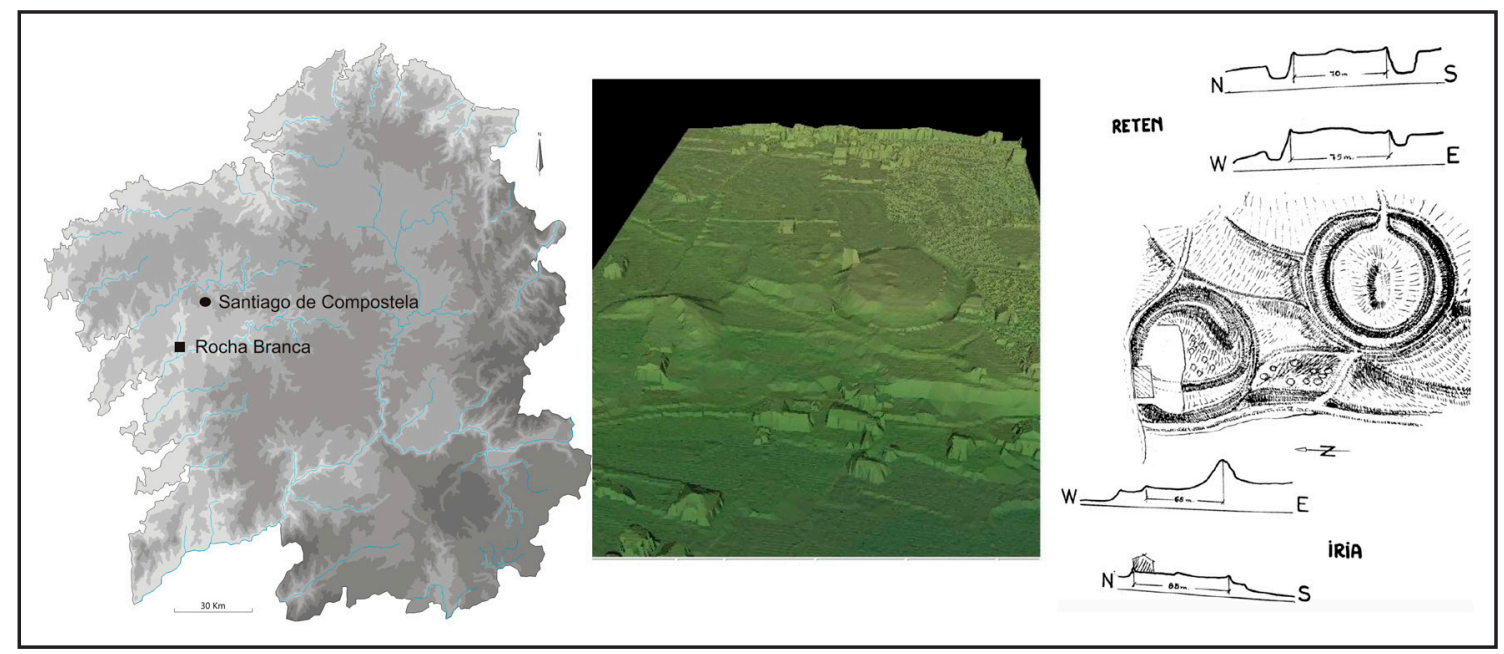

Figura 1. Mapa de localización del yacimiento, MTD (a partir de Patrimonio Galego) y detalle del yacimiento (a partir de FERNÁNDEZ ABELLA, 2015).

\section{Planteamientos y ObJetivos}

Como se podrá observar, el material disponible para su estudio está muy fragmentado, y estas condiciones no permiten una reconstrucción de las piezas. Sin embargo, algunas piezas recuperadas completas a principios del siglo XX en A Rocha Branca han sido depositadas en el Museo de Cáceres en 1945, y recientemente publicadas (FRANCO, 2013). En dicho estudio se considera que estas piezas son encargadas por el arzobispo de Mendoza a talleres valencianos, formando parte de una dinámica de la aristocracia y el clero generalizada en Europa desde el siglo XIII, consistente en adornar sus viviendas con azulejos valencianos como una forma de ostentación y lujo (FRANCO, 2013).

La bibliografía sobre azulejería en arqueología es muy amplia en la España mediterránea. De hecho, la producción valenciana es bien conocida, considerándose que en el siglo XIII y XIV se basa en una tradición mediterránea y del mundo musulmán, lejos de la influencia de las producciones europeas (COLL, 2009: 97). Este estilo se exportó más allá del área de influencia catalano-aragonesa, alcanzando Castilla, Italia y otros países europeos, entrando en decadencia a la vez que el Reino de Valencia se convierte en un área periférica a lo largo del siglo XVI (ALGARRA, 1998: 154).

El proceso de producción completo es bien conocido, ya que hay fuentes documentales que hablan de la entrega de plomo y estaño² a los alfareros de la región o encargos concretos de fabricación de personas relevantes de la época como Pedro el Ceremoniosos en 1367 para Tortosa y Barcelona (COLL, 2009: 99) entre muchos otros encargos bien documentados.

Entre los azulejos decorados a pincel podemos destacar la introducción del azul cobalto en el primer tercio del s. XIV. El cual conseguirá relegar a segundo plano el azulejo monocromo en menos de cien años, usándose una base de esmalte estannífero y pigmen-

2 Composición también encontrada en los vidriados de la cerámica sevillana del siglo XIV (LAFUENTE, 2010). 
tos de óxido de cobalto, magnesio y a veces cobre en oxidación, así mismo la decoración es inspirada en temas musulmanes (COLL, 2009: 103). En los inicios del siglo XV, las composiciones azules se desarrollan con motivos florales (de puntos, de perejil, zarcillos con hojitas) y epigráficos de la serie AVE MARIA (COLL, 2009: 103), que se traducen en motivos vegetales esquematizados y geométricos (ALGARRA, 2000), entre otros.

Entre los muchos encargos conocidos a los talleres valencianos, se considera el del obispo don Lope de Mendoza c. 1407, 'fecha aproximada de los azulejos con emblemas compostelanos formados por el sombrero de peregrino y la venera, junto a la inscripción 'Senthiago' del palacio Rocha de Padrón' (COLL, 2009: 106). Los azulejos de A Rocha Branca, pertenecen a la serie de temas heráldicos, en particular al grupo de identificación personal o grupal, presentando una figura que identifica una institución, en este caso religiosa, desde donde se impondría el criterio de la decoración al alfarero.

Sin embargo, dada la tradición azulejera de la ciudad del Guadalquivir en época medieval (DOMÍNGUEZ, 1998) y dado el origen sevillano del obispo, se ha cuestionado que su encargo fuera realmente a talleres valencianos (VILLA-AMIL, 1907), o incluso el mismo autor ha especulado con un posible origen toledano (VILLA-AMIL, 1907:36). Si bien es cierto que los estilos documentados, de los azulejos figurativos, tienen claros paralelos con azulejos valencianos y no con sevillanos.

El objetivo de este trabajo es caracterizar desde un punto de vista formal y composicional, una muestra representativa de las piezas del yacimiento para poder valorar la procedencia de sus materiales. En la medida de lo posible, se tratará de comprobar si las materias primas son compatibles con las existentes en Galicia o por el contrario se puede apuntar hacia un origen foráneo, en especial valenciano o sevillano.

\section{Materiales y Metodología}

El estudio que presentamos forma parte de un conjunto de piezas depositadas en el Departamento de Historia del Departamento de Santiago de Compostela. Se ha realizado una selección representativa de los tipos de piezas asociadas al material constructivo, compuesto por 488 piezas, procedente de la mencionada intervención de 1981. Dentro de este conjunto 90 son azulejos, 23 son fragmentos de material constructivo variados asociados al revestimiento de las paredes y el resto son teselas cerámicas con dos tamaños estandarizados, el tamaño mayoritario recuperado es pequeño, rondando los 30-35 $\mathrm{mm}^{2}$ (257 piezas) y las restantes rondan los $70 \mathrm{~mm}^{2}$.

Dado que el conjunto analizado está conformado por un número de piezas representativo, pero pequeño, realizaremos una descripción pieza a pieza como primer paso del trabajo, combinando una descripción tanto de las características físicas observadas como de la composición de las piezas.

En el estudio arqueométrico se analiza el cuerpo cerámico a través de las técnicas de difracción de rayos X de polvo cristalino (DRX) para identificar la mineralogía y de espectrometría de fluorescencia de rayos $\mathrm{X}(\mathrm{XRF})$ para determinar la composición elemental. En los fragmentos escogidos para el análisis, se retira cuidadosamente el vidriado 
y se muele el cuerpo cerámico hasta un tamaño de partícula de $50 \mu \mathrm{m}$ que, después de homogeneizado, es el utilizado para el análisis mineralógico y elemental según las condiciones de medida que se detallanen MARTÍNEZ CORTIZAS et al. (2008). Los vidriados se micromuestrean y se analizan en microscopía electrónica de barrido acoplada con una microsonda de rayos X (SEM-EDX) para estudiar su morfología y determinar su composición elemental. Para ello, se seleccionan esquirlas de los diferentes vidriados (según su color) que se limpian con acetona y se depositan en un porta de microscopía electrónica. Las esquirlas se analizan bajo vacío, sin sombrear y se recogen imágenes y los espectros EDX según se desarrolla en un trabajo recientemente publicado (LANTES et al., 2011).

\section{Resultados}

En este apartado realizaremos una descripción detallada de las seis piezas y de los resultados arqueométricos. Éstos reflejan los datos analíticos sintetizados en las tablas del apartado Discusión y conclusiones.

RO/81 F-5 (RBo1). Fragmento esquinal de un azulejo decorado, en esmalte blanco, blanco azulado y azul. La textura es compacta y bastante bien decantada y la fractura es bícroma, sepia con interior amarillento. El diseño se distribuye en cuadrados y rectángulos delimitados por líneas finas azules, el desconchado sólo permite apreciar el dibujo de una flor con 4 pétalos, que se corresponde posiblemente con la parte inferior derecha del esquinal. El vidriado presenta varios desconchados y no conserva esmalte en la superficie interna pero sí en un lateral que nos permite pensar que es un fragmento próximo al remate o esquinal. El fragmento mide 57x66×18 mm, y forma parte de la misma decoración que la pieza $\mathrm{RO} / 81 \mathrm{~F}-3$ 40, ambos muestran el mismo programa iconográfico.

Los minerales que se encuentran en el cuerpo cerámico son cinco: cuarzo, feldespato potásico, diópsido, gehlenita y hematita. La gehlenita es mayoritaria. Los tres últimos minerales citados son indicadores de procesos de alta temperatura (LINARES et al., 1991). Por su presencia, estimamos que las cerámicas debieron cocerse en un rango de temperaturas de $900-1100{ }^{\circ} \mathrm{C}$, pero no superior puesto que se habría destruido la gehlenita y sin embargo esta es abundante. La composición elemental del cuerpo cerámico es acorde con la mineralogía identificada. En especial, la alta concentración de calcio $(13,9 \%)$ y relativamente alta de magnesio $(1,2 \%)$, son coherentes con la presencia de gehlenita y de diópsido, pues estos minerales se forman en alta temperatura si hay calcio y magnesio abundantes. Se puede deducir que los tipos de materia prima con el que se elaboraron estas cerámicas tuvieron que ser arcillas ricas en calcio y magnesio, esto es, arcillas calizo-dolomíticas, quizás margas.

Sobre el vidriado se realizaron varias submuestras. En una submuestra de esquirla de vidriado azul se detecta plomo (23,3\%) pero con presencia también de otros fundentes en menor proporción como el sodio, magnesio, potasio y calcio. La presencia de cloro en proporción estequiométrica al sodio, podría indicar la adición de sal en 
la fórmula del vidriado. El color azul está causado por la presencia de cobalto y cobre. En algunas áreas se encuentran ocasionalmente pequeñas manchas homogéneas (grises en color relativo SEM) y de forma ovalada. En un análisis, se comprobó que su composición es fundamentalmente silicio y oxígeno, indicando que se trata de granos de cuarzo que quizás no se fundieron homogéneamente en el proceso de formación del vidriado. En otra submuestra de vidriado blanco-azulado se comprobó como su composición es similar al vidriado azul, si bien, no se detectan cobalto y cobre. Finalmente se analizó una esquirla de vidriado blanco. Su composición es similar a la del vidriado blanco-azulado.

RO/81 F-3 40 (RBO2). Fragmento de azulejo decorado, en esmalte blanco y azul, se representa un esquinal, con dibujo de una flor. El vidriado presenta varios desconchados laterales y se conserva en el esquinal y ambas superficies, exterior e interior. La pasta es sepia y la fractura bicroma, sepia con interior amarillento, bastante bien decantada con textura compacta. Sus medidas son: 40x35x14 mm. Forma parte de la misma decoración que la pieza anterior $\mathrm{RO} / 81 \mathrm{~F}-5$.

El cuerpo cerámico, al ser similar al del azulejo anterior, no fue analizado, así que remitimos a los datos de RBo1. Sin embargo, el vidriado azul de esta muestra, sí analizado, contiene plomo como principal fundente además de algo de sodio, magnesio, calcio y potasio. En esta ocasión no se detecta cloro, como sucedió con el azulejo RBo1. Los elementos químicos que aportan el color azul son cobalto, níquel y cobre. El níquel no se detectó tampoco en la muestra anterior. Se detecta estaño, que es un elemento opacificador en esmaltes. La base del vidriado (cuerpo cerámico) es más pobre en silicio y más rica en hierro pero se detectan igualmente cobre, níquel, estaño y plomo, que sugiere una difusión de estos elementos químicos hacia la interfase vidriado-cuerpo cerámico. El vidriado blanco (o blanco azulado) es similar al vidriado azul, únicamente puede diferenciarse por la ausencia de los elementos químicos colorantes, si bien se detectan trazas de cobre, que podrían estar causando el ligero tono azulado del mismo.

RO/81 F-9 4-50 (RBo3). Fragmento de azulejo de forma rectangular (62x51x17 mm). Posee pastas rojas de texturacompacta y fina, bien decantadas. El vidriado es de color marrón oscuro casi negro, bien conservado en el exterior y laterales, mientras que ha desaparecido de su cara interna y de los laterales.

Los minerales que se encuentran en esta cerámica son cinco: cuarzo, feldespato potásico, plagioclasa, diópsido y hematita. La plagioclasa es el mineral mayoritario. La presencia de diópsido orienta un rango de temperaturas de cocción del orden de los 900-1100 ${ }^{\circ} \mathrm{C}$. La composición elemental del cuerpo cerámico es acorde con la mineralogía identificada. En especial, la concentración relativamente alta de magnesio $(1,8 \%)$ es coherente con la detección del mineral diópsido, según se indicó para la muestra anterior. La ausencia de gehlenita ayuda a concretar el intervalo de temperaturas de cocción, éste mineral cristaliza en la cocción en estos materiales si hay calcio abundante, como es el caso en esta muestra (5,0\%), y se destruye sobre los $1000{ }^{\circ} \mathrm{C}$ 
(LINARES et al., 1983). Se puede deducir pues que la temperatura de cocción ha superado ligeramente $\operatorname{los} 1000{ }^{\circ} \mathrm{C}$. La combinación de minerales y elementos químicos apunta a que las materias primas con las que se elaboraron estas cerámicas pudiesen ser arcillas ricas en calcio y magnesio, esto es, arcillas calizo-dolomíticas quizás margas.

El vidriado de este azulejo presenta un color marrón homogéneo. Está ligeramente rayado en su superficie. Contiene plomo como fundente y con pequeñas cantidades de sodio, magnesio, potasio y calcio, éste último en mayor proporción. Se identifica además hierro y manganeso con una función posiblemente colorante. La superficie no vidriada tiene una composición bastante similar a la vidriada, aunque el contenido de silicio es menor, así como el de calcio, manganeso y plomo.

RO/81 B-2 80 (RBO4). Azulejo de forma cuadrada, con una esquina rota aunque parece su tamaño original $(70 \times 70 \times 15 \mathrm{~mm})$. Presenta pastas sepias homogéneas en fractura y texturas harinosas finas. El vidriado exterior es de color blanco verdoso, pero en su interior apenas conserva superficie vidriada.

Los minerales que se encuentran en el cuerpo cerámico de esta pieza son cuatro: cuarzo, feldespato potásico, plagioclasa y diópsido. El cuarzo es el mineral mayoritario y la plagioclasa el minoritario. La presencia de diópsido y su abundancia, así como la ausencia de gehlenita (la pasta tiene calcio: 4,4\%) sugiere, al igual que se apuntó para la muestra anterior, una temperatura de cocción ligeramente superior a los $1100^{\circ} \mathrm{C}$. La composición apunta a la utilización de arcillas calizo-dolomíticas, quizás margas.

Este azulejo tiene un vidriado en la cara externa de base blanca con un ligero tono verdoso. Se encuentra relativamente picado en superficie. Un análisis puntual en el vidriado revela presenciade plomo como fundente además de cierta cantidad de sodio y de calcio. Destaca la además presencia de estaño como elemento opacificador. En otro análisis de área se comprueba una composición similar con magnesio, fósforo y cloro adicionales. El análisis de la superficie no vidriada es similar al del vidriado, destacando, eso sí, menores niveles de silicio. También se detecta algo de estaño y plomo lo que indicaría difusión de estos elementos hacia el cuerpo cerámico.

RO/81 H-4 50 (RBo5). Fragmento de azulejo rectangular (66x31X15 mm). La pasta es amarillenta y bien decantada de texturas harinosas finas. Posee un vidriado de color verde apagado, está algo desconchado en un esquinal exterior y su conservación en la cara interna es meramente testimonial.

Los minerales que se identifican en esta cerámica son seis: cuarzo, plagioclasa, calcita, diópsido, hematita y analcima. El diópsido es el mineral mayoritario, seguido del cuarzo. La presencia de diópsido y la ausencia de gehlenita (a pesar de analizarse niveles de calcio del del 11,19\%) sugieren que la temperatura de cocción ha debido de ser ligeramente superior a $\operatorname{los} 900-1100^{\circ} \mathrm{C}$, al igual que en las dos muestras anteriores. También se detecta calcita, mineral que se descompone térmicamente a los 800$900{ }^{\circ} \mathrm{C}$. La presencia de calcita puede parecer contradictoria pero lo más probable es 
que se trate de calcita neoformada post-deposicionalmente. Este hecho está apoyado por la detección adicional de analcima, una zeolita que aparece habitualmente porneoformación post-deposicional (SCHWEDT et al., 2006). En cuanto a la composición elemental del cuerpo cerámico, hay coherencia con la mineralogía identificada, en especial la concentración relativamente alta de magnesio concuerda con la presencia de diópsido. Destaca también la alta concentración de hierro encontrada así como la alta cantidad de plomo, originada probablemente por difusiones desde el vidriado. La composición, como en los casos anteriores apunta a la utilización de arcillas calizo-dolomíticas quizás margas. Habría que destacar un ambiente post-deposicional húmedo que facilitaría la formación de minerales secundarios como la analcima y la calcita.

En un análisis efectuado sobre la superficie del vidriado (en esta muestra se analiza la pieza entera en SEM sin micromuestreo) se identifica plomo con cantidades relativamente considerables de calcio y menores de sodio y de magnesio (todos ellos, fundentes). El color es aportado por el cobre y el agente opacificante es el estaño.

RO/81 D-2 35 (RBo6). Material constructivo cerámico, forma rectangular con una gran pestaña, es una pieza interpretada como el marco de una ventana o puerta (140x57x40 mm). Presenta un grado de rodamiento elevado en su parte interior. En dos de sus caras presenta vidriado verdoso apagado. Su pasta es amarillenta y bien decantada de texturas harinosas finas.

Los minerales que se encuentran en esta cerámica son únicamente cuarzo y diópsido. El diópsido es el mineral mayoritario. La presencia de diópsido, como en los casos anteriores expuestos indica un rango de temperaturas de cocción del orden de los 900$1100{ }^{\circ} \mathrm{C}$. La ausencia de cualquier otro mineral primario del tipo micas, feldespatos, plagioclasas o bien secundario, p. ej. del tipo gehlenita (que se forma en presencia de calcio y en esta muestra hay abundancia del mismo con un 6,64\%) indica que es muy probable que las temperaturas alcanzadas en la cocción sean del orden de los 1100 ${ }^{\circ} \mathrm{C}$ o incluso algo superiores. La composición elemental es acorde con la mineralogía identificada. En especial, la concentración relativamente alta de magnesio es coherente con la presencia del diópsido, como en casos anteriores. Destaca igualmente la alta concentración de hierro y los niveles de plomo detectados, que probablemente se deban a contaminaciones por el vidriado. Las materias primas utilizadas, como para los otros casos, serían arcillas calizo-dolomíticas, quizás margas.

El vidriado de este esquinal constructivo contiene plomo como fundente con cantidades relativamente abundantes de calcio y menos abundantes de sodio y de magnesio. El color es aportado por el cobre. Se detecta estaño como agente opacificante. La composición del vidriado es, a grandes rasgos, similar a la de la muestra anterior, RBo5. 


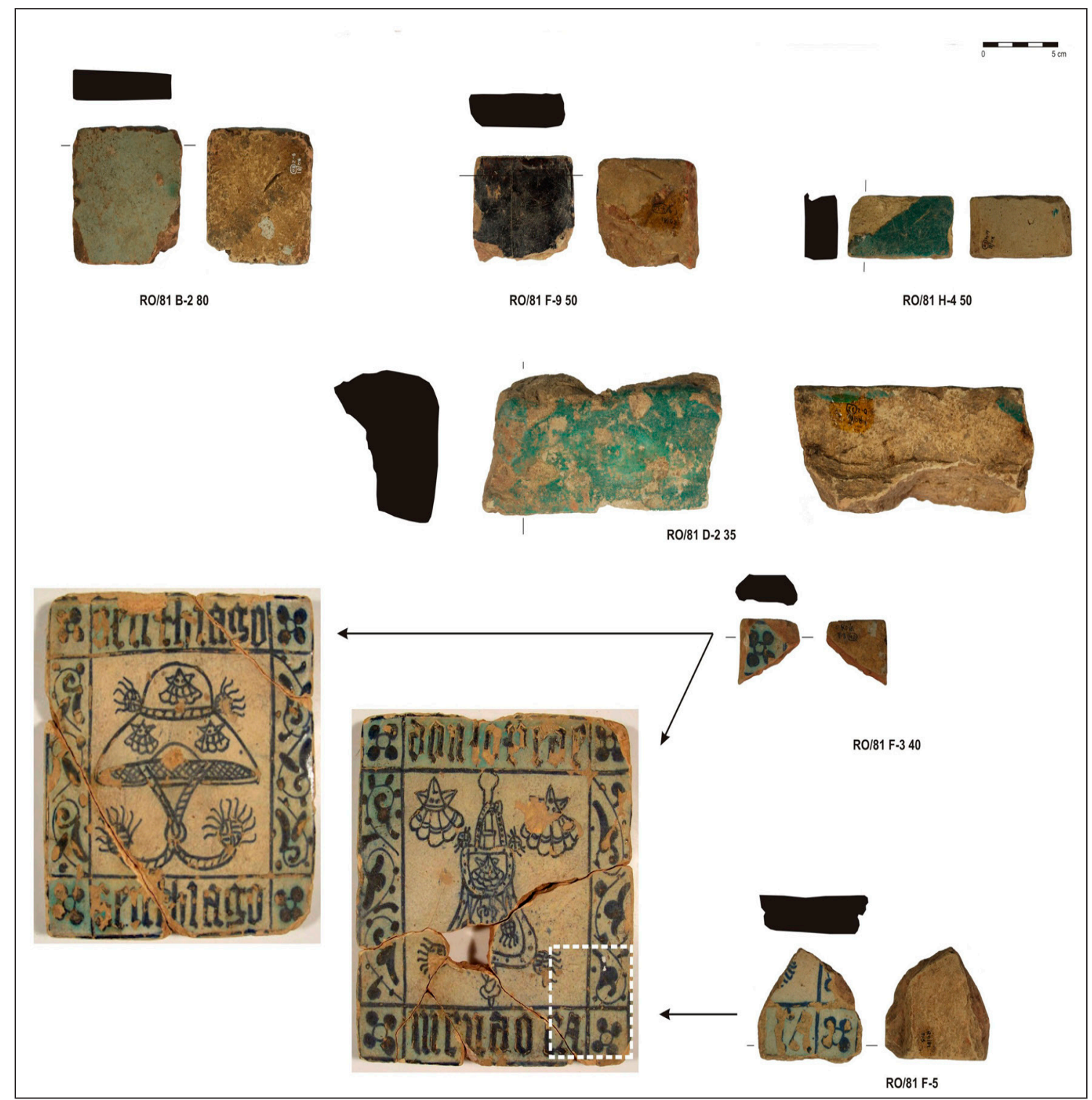

Figura 2. Fotografía de los fragmentos analizados. Los 2 azulejos completos utilizados como muestra completa están depositados en el Museo de Cáceres (tomado a partir de FRANCO, 2013).

Tabla 1. Mineralogía del cuerpo cerámico de los azulejos. Datos en \% de la fase cristalina.

\begin{tabular}{lccccccccc}
\hline$\%$ & Analcima & Mullita & Cuarzo & Feldespato K & Plagioclasa & Calcita & Diópsido & Gehlenita & Hematita \\
RB01 & - & - & 17 & 3 & - & - & 16 & 60 & 3 \\
RB03 & - & - & 27 & 11 & 31 & - & 17 & - & 14 \\
RB04 & - & - & 48 & 4 & 7 & - & 41 & - & - \\
RB05 & 5 & - & 29 & - & 3 & 12 & 46 & - & 5 \\
RB06 & - & - & 44 & - & - & - & 56 & - & - \\
\hline
\end{tabular}


Tabla 2. Composición elemental del cuerpo cerámico de los azulejos. u: unidades.

\begin{tabular}{|c|c|c|c|c|c|c|c|c|c|c|c|c|c|c|}
\hline & C & $\mathbf{N}$ & $\mathrm{Mg}$ & Al & Si & $\mathbf{P}$ & $S$ & $\mathrm{Cl}$ & K & $\mathrm{Ca}$ & $\mathrm{Ti}$ & $\mathrm{Fe}$ & $\mathrm{Cr}$ & $\mathrm{Ba}$ \\
\hline RB01 & 1,35 & 0,01 & 1,22 & 8,8 & 21,2 & 0,06 & 0,00 & 0,08 & 1,15 & 13,90 & 0,28 & 3,27 & 39 & 36 \\
\hline RB02 & & & - & - & - & - & - & - & - & - & - & - & - & - \\
\hline RB03 & 0,97 & 0,04 & 1,78 & 10,9 & 28,6 & 0,03 & 0,00 & 0,05 & 1,85 & 4,99 & 0,49 & 5,04 & 90 & - \\
\hline RB04 & 1,36 & 0,10 & 1,85 & 10,6 & 23,9 & 0,34 & 0,05 & 0,07 & 1,88 & 4,38 & 0,46 & 4,88 & 101 & 369 \\
\hline RB05 & 1,84 & 0,04 & 2,19 & 7,8 & 23,3 & 0,22 & 0,00 & 0,06 & 0,93 & 11,19 & 0,31 & 4,19 & 64 & 457 \\
\hline RB06 & 1,14 & 0,07 & 1,93 & 10,5 & 24,6 & 0,11 & 0,02 & 0,06 & 1,56 & 6,64 & 0,38 & 4,22 & 80 & 267 \\
\hline u & $\%$ & $\%$ & $\%$ & $\%$ & $\%$ & $\mathrm{ppm}$ & $\mathrm{ppm}$ & ppm & $\%$ & $\%$ & $\%$ & $\%$ & ppm & $\mathrm{ppm}$ \\
\hline & $M n$ & $\mathrm{Ni}$ & $\mathrm{Cu}$ & $\mathrm{Zn}$ & Ga & As & $\mathrm{Br}$ & $\mathbf{R b}$ & $\mathrm{Sr}$ & $Y$ & $\mathrm{Zr}$ & Nb & $\mathbf{P b}$ & Th \\
\hline RB01 & 454 & 31 & 85 & 68 & - & - & - & 99 & 258 & 64 & 199 & 17 & 1587 & - \\
\hline RB02 & - & - & - & - & - & - & - & - & - & - & - & - & - & - \\
\hline RB03 & 1076 & 48 & 53 & 82 & - & 12 & 9 & 101 & 218 & 53 & 353 & 20 & 735 & - \\
\hline RB04 & 1051 & 47 & 61 & 107 & - & - & 12 & 94 & 191 & 78 & 245 & 19 & 2125 & - \\
\hline RB05 & 797 & 42 & 822 & 86 & - & - & - & 117 & 364 & 120 & 199 & 17 & 5152 & - \\
\hline RB06 & 848 & 51 & 70 & 84 & - & 13 & 11 & 104 & 309 & 51 & 222 & 21 & 908 & 3 \\
\hline u & ppm & ppm & ppm & $\mathrm{ppm}$ & $\mathrm{ppm}$ & $\mathrm{ppm}$ & $\mathrm{ppm}$ & ppm & ppm & ppm & ppm & $\mathrm{ppm}$ & ppm & $\mathrm{ppm}$ \\
\hline
\end{tabular}

Tabla 3. Síntesis de la composición elemental de los vidriados, a partir del análisis EDX.

\begin{tabular}{|c|c|c|c|c|c|c|}
\hline & Color & Red & Estabilizante & Fundente & Opacificador & Colorante \\
\hline \multirow[t]{2}{*}{ RB01 } & blanco & Si & $\mathrm{Al}$ & $\mathrm{Pb},(\mathrm{Na}, \mathrm{K}, \mathrm{Ca}, \mathrm{Mg})$ & - & - \\
\hline & azul & $\mathrm{Si}$ & $\mathrm{Al}$ & $\mathrm{Pb},(\mathrm{Na}, \mathrm{K}, \mathrm{Ca}, \mathrm{Mg})$ & - & $\mathrm{Co}, \mathrm{Cu}$ \\
\hline \multirow[t]{2}{*}{ RB02 } & blanco & $\mathrm{Si}$ & $\mathrm{Al}$ & $\mathrm{Pb},(\mathrm{Na}, \mathrm{K}, \mathrm{Ca}, \mathrm{Mg})$ & - & - \\
\hline & azul & $\mathrm{Si}$ & $\mathrm{Al}$ & $\mathrm{Pb},(\mathrm{Na}, \mathrm{K}, \mathrm{Ca}, \mathrm{Mg})$ & Sn & $\mathrm{Co}, \mathrm{Cu}, \mathrm{Ni}$ \\
\hline RB03 & marrón & Si & Al & $\mathrm{Pb},(\mathrm{Ca}, \mathrm{K})$ & - & $\mathrm{Mn}, \mathrm{Fe}$ \\
\hline RB04 & blanco & $\mathrm{Si}$ & $\mathrm{Al}$ & $\mathrm{Pb},(\mathrm{Na}, \mathrm{Mg})$ & Sn & - \\
\hline RB05 & verde & Si & $\mathrm{Al}$ & $\mathrm{Pb},(\mathrm{Ca})$ & Sn & $\mathrm{Cu}, \mathrm{Fe}$ \\
\hline RB06 & verde & $\mathrm{Si}$ & $\mathrm{Al}$ & $\mathrm{Pb},(\mathrm{Ca})$ & Sn & $\mathrm{Cu}, \mathrm{Fe}$ \\
\hline
\end{tabular}




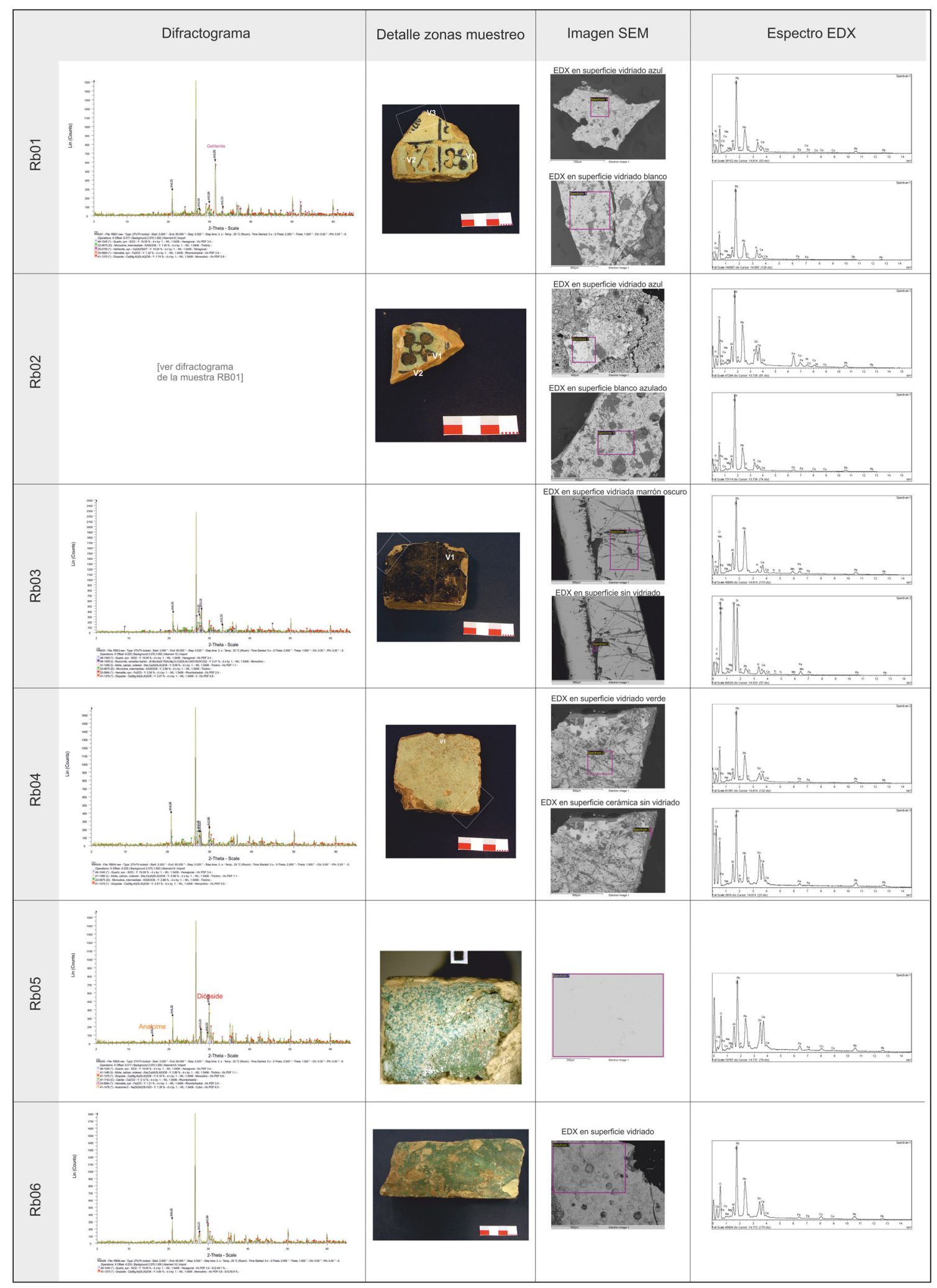

Figura 3. Resultados analíticos de las piezas. 


\section{Discusión}

En relación con los análisis de las piezas (tabla 1, tabla 2 y figura 3), encontramos que los cuerpos cerámicos de los azulejos de Rocha Branca contienen casi siempre cuarzo y diópsido. Los feldespatos potásicos están presentes en tres muestras RBo1, RBo3 y RBo4 y la plagioclasa en RBo3, RBo4 y RBo5. También se detecta hematita en algunas muestras. La presencia de diópsido se debe a la neoformación en el proceso de cocción de las pastas (entre $800-900$ y $1100{ }^{\circ} \mathrm{C}$ o incluso algo más) si hay calcio y magnesio abundante en las pastas. La gehlenita detectada en RBo1 es también un mineral de neoformación (formado en presencia de calcio) en un rango de temperaturas similar, si bien se destruye antes de los $1100{ }^{\circ} \mathrm{C}$. La combinación por presencia/ausencia de estos minerales indicadores, sugieren pues que las temperaturas de cocción tuvieron que sobrepasar ligeramente los $1100{ }^{\circ} \mathrm{C}$ en la mayoría de los azulejos, salvo en RBo1 que no debió de superar este rango. Estas temperaturas, determinadas por la presencia de estos minerales indicadores es la característica que se requiere para conseguir una buena fusión de los vidriados de plomo, en especial si la concentración de este fundente no es muy elevada (TITE et al., 1998).

Como indicadores de una fase de abandono en un ambiente húmedo, aparecen la analcima y la calcita detectadas en RBo5, coherente con las condiciones de humedad del yacimiento de Rocha Branca.

Los vidriados de los azulejos son todos ellos de base sílice, con aluminio como estabilizador y plomo como fundente (tabla 3). Como fundentes hay además cantidades pequeñas y variables de otros elementos químicos alcalinos y alcalinotérreos. El elemento opacificador es siempre el estaño. Los colorantes utilizados son habitualmente el cobalto, cobre y níquel para los azules, manganeso e hierro (en ocasiones también cromo) para los marrones y cobre e hierro (en ocasiones también níquel y cromo) para los verdes.

En cuanto al tipo de materia prima con la que se han elaborado estas pastas, los minerales identificados y la composición elemental apuntan a que se pueda tratar de arcillas caolinítico-calcáreas o margosas. En cualquier caso la composición de los cuerpos cerámicos es muy similar entre sí (incluso las temperaturas de cocción son similares) lo que haría factible pensar en un centro productor común, eso sí, ubicado fuera de Galicia, pues no se dan este tipo de materias primas en este territorio.

La composición de las pastas de los cuerpos cerámicos de estos azulejos, es muy similar a la de la de cerámicas de Manises, Paterna y de Sevilla según datos analizados por POLVORINOS DEL RÍO Y CASTAING (2010); POLVORINOS et al. (2011) y ROMEROPASTOR et al. (2015), y podrían tener su origen allí. Es difícil discernir entre un origen sevillano o valenciano, por la similitud de las concentraciones de los elementos químicos entre todos ellos, tanto para los elementos mayoritarios como para los trazas. Si bien, si se comparan en detalle nuestros datos con los datos proporcionados por POLVORINOS DEL RÍO Y CASTAING (2010) y POLVORINOS et al. (2011) se constata como para algunos elementos químicos como el aluminio, rubidio, estroncio e itrio los valores de los azulejos de Rocha Branca son prácticamente idénticos a los valores de las cerámicas de Valencia (en especial Paterna) y algo más diferentes de los de Sevilla, aunque hay que 
también destacar que nuestros valores para el calcio son inferiores a los de ambas áreas productoras en el caso de Rbo3, Rbo4 y Rbo6.

Asimismo, la composición de los vidriados en todos los casos, también es similar a estos mismos centros productores, con base de silicio, plomo y alcalinos (en especial potasio) como fundentes, y presencia de estaño como opacificador en el caso de los esmaltes. Si bien, también hay que mencionar que este tipo de vidriados plomados y esmaltados con estaño eran muy frecuentes en diferentes tipos cerámicos de la Edad Media y Moderna, islámicos, mayólicas de centros productores de Teruel, Cataluña, Sevilla, Italia e incluso oriente (COMODI et al., 2004; IÑÑNEZ et al., 2009; GIANONCELLI et al., 2008; MASON et al., 1997; MOLERA et al., 2001; MOLERA et al., 2005; PÉREZ-ARANTEGUI et al., 2005; RICCI et al., 2005) por lo que el carácter discriminador del tipo de vidriado para el origen es relativo.

En cualquier caso, y con seguridad, se puede concluir que son materiales constructivos fabricados fuera de Galicia, o al menos a partir de materias primas foráneas, puesto que este tipo de arcillas de carácter calcáreo-margoso no están presentes en el Noroeste Peninsular, donde las materias primas arcillosas más frecuentes son las de tipo caolinítico no calcáreo derivadas de la alteración de granitos y de esquistos. Y como se indicó anteriormente, se sugiere un posible origen valenciano para las mismas.

Desde un punto de vista estilístico, las piezas decoradas sí responden a un estilo plenamente valenciano, como muchos autores han apuntado con anterioridad (ALGARRA, 2000; COLL, 2009; FRANCO, 2013), el parecido estilístico con azulejos del inventario del MNCASGM (n. ${ }^{\circ}$ de inventario CE1/o2246, CE1/o2233, CE1/o2258, CE1/o2259, CE1/o2467, CE1/o2512 (MNCASGM, base de datos Ceres) no es encontrado en los azulejos sevillanos por el momento. Entre los azulejos sevillanos no se identifica mayoritariamente el azul sobre blanco y si otro tipo de colores, como verdes, naranjas y negros, así como otras técnicas como la cuerda seca o la arista (DOMINGUEZ 1998:141). El verde y el marrón casi negro chocolate son los colores predominantes de las otras piezas documentadas en A Rocha Branca. Por lo que podríamos plantear como hipótesis que quizás no toda la producción azulejera fuera encargada en un único taller, y, además de Valencia pudiera haberse encargado en talleres sevillanos también.

\section{Conclusiones}

Si bien a nivel documental es más complicado dilucidar el origen de estos azulejos, el estilo de los mismos es claramente valenciano, correspondiéndose con otras piezas de Manises realizadas en el siglo XV, quedando el estilo de los azulejos monocromos con más dudas por el momento. Desde el punto de vista arqueológico, las piezas estudiadas, son representativas del lujo propio de un castillo de la mitra compostelana, respondiendo a un estilo cerámico que encaja bien en la tradición valenciana de encargos suntuosos que condicionan la iconografía de las piezas. Sin embargo, las características técnicas de las pastas, y del propio vidriado, nos llevan a un foco productor de tradición alfarera mudéjar, que no nos permite concretar más el posible foco productor, aún encajando 
con centros valencianos y sevillanos. Sin más documentación, únicamente estos análisis nos permiten confirmar su fabricación foránea, a lo que se suma la falta de constancia de lugares de producción de material constructivo en cerámica en Galicia, salvo de teja.

Deseamos resaltar que con este trabajo intentamos contribuir al conocimiento más detallado de un tipo de material cerámico todavía poco estudiado en el noroeste peninsular, y que es interesante conocer no sólo desde el punto de vista estilístico sino también analítico. Pocos restos materiales se conservan de aquella pequeña intervención arqueológica realizada en el yacimiento de Rocha Branca, y es la primera vez que este tipo de elemento arqueológico se analiza. Podemos confirmar con los análisis, la procedencia foránea de estos materiales, compatible con un posible origen valenciano. Sin embargo, son necesarias otro tipo de analíticas, como los análisis isotópicos, para matizar la procedencia de forma más concreta. Poco a poco vamos sumando investigaciones parciales a los estudios ya realizados sobre el yacimiento con la esperanza de ir incorporándolos a un puzzle en el que todavía quedan muchas piezas por encajar.

\section{Reconocimientos}

Este trabajo ha sido desarrollado en el marco de 2 proyectos:

"Isótopos de Pb e Sr en cerámicas arqueológicas de Galicia: estudio de la procedencia y el acceso a las materias primas" (EM 2012/054) (2012-PG217), financiado por Ayudas a Proyectos de Investigación a Investigadores Emergentes del Plan Gallego de I+D+I, Consellería de Cultura, Educación e Ordenación Universitaria entre 2012 y 2015.

"Tecnología y producción de la cerámica medieval de Galicia" (MC-PTG). HAR201564441-P (Plan Nacional: Ministerio de Economía y Competitividad, Convocatorias 2015, Proyectos EXCELENCIA y Proyectos RETOS, Dirección General de Investigación Científica y Técnica, Subdirección General de Proyectos de Investigación), 2016-2019.

El procesado de la muestra y los análisis fueron realizados en el año 2015 por Marta Pedrouzo Piñeiro y Uxía Iglesias Álvarez en la Unidad de Arqueometría (RIAIDT) de la Universidad de Santiago de Compostela dentro el marco de las Prácticas Académicas Externas del máster de Arqueología y Ciencias de la Antigüedad de la USC bajo la dirección de Oscar Lantes Suárez.

\section{Bibliografía}

ALGARRA PARDO, V. M. 1998. "Azulejería gótica valenciana. Canal de mensajes de identificación social (estilo, espacios y usuarios)”. En J. I. Padilla y J. M. Vila (cords.), Ceràmica medieval i postmedieval. Circuits productius i seqüencies culturals. Monografies d'Arqueologia Medieval I Postmedieval 4. Barcelona, pp. 145-163.

ALGARRA PARDO, V. M. 200o. "Azulejería bajomedieval y tardomedieval valenciana (siglos XIIIXV)”. En La ruta de la cerámica. Sala Bancaja San Miguel. Castellón, pp. 66-73.

CARAMÉS MOREIRA, V., CASTRO LORENZO, M. L., SUÁREZ OTERO, J. 2006. "Cerámicas de lujo en la Galicia del siglo XV”., En Os Capítulos da Irmandade. Peregrinación y conflicto social en la Galicia del siglo XV. Tórculo Artes Gráficas. Santiago de Compostela, pp. 200-211. 
CASAMAR, M. 1979. "Colección de azulejos del Museo de Pontevedra”, El Museo de Pontevedra, 33, 331-347. https://doi.org/10.3989/ceg.1992.v40.i105.303

COLL CONESA, J. 2009. La cerámica valenciana (apuntes para una síntesis). Asociación Valenciana de Cerámica. Ribarroja del Túria.

COMODI, P., BERNARDI, M., BENTIVOGLIO, A., GATTA, G. D., ZANAZZI, P. F. 2004. "The production and technology of glazed ceramics from the middle ages, found in the Saepinum Territory (Italy): a multimethodic approach", Archaeometry, 46(3): 405-419. https://doi.org/10.1111/ j.1475-4754.2004.00165.x.

DOMÍNGUEZ CABALLERO, R. $\mathrm{M}^{\mathrm{a}}$. 1998. "Evolución del azulejo sevillano desde el siglo XIII. Técnicas.” En F. Bores, J. Fernández, S. Huerta y E. Rabasa (coords.) Actas del Segundo Congreso Nacional de Historia de la Construcción. A Coruña, pp. 139-144.

FERNÁNDEZ ABELLA, D. 2015. "El castillo de A Rocha Branca de Padrón (A Coruña)", Férvedes, 8: 411-419.

FRANCO POLO, N. M. 2013. "Azulejos decorados con motivos jacobeos Castillo de la Rocha Blanca (Padrón, A Coruña) Siglo XV”, Pieza del mes de Julio de 2013. Museo de Cáceres (http://museodecaceres.blogspot.com.es/2013/o7/la-pieza-del-mes-julio-de-2013.html (consultado el 17 de marzo de 2016). https://doi.org/10.25176/rfmh.v17.n4.1211

GIANONCELLI, A., CASTAING, J., BOUQUILLÓN, A., POLVORINOS, A., WALTER, P. 2008. “Análisis cuantitativo de la composición de los vidriados de Della Robbia de Sevilla con un espectrómetro portátil de Fluorescencia de Rayos X". En: S. Rovira Llorens, M. García Heras, M. Gener Moret e I. Montero Ruiz (eds.), Actas del VII Congreso Ibérico de Arqueometría, CSIC, Museo Arqueológico Nacional, Madrid, pp. 309-318. https://doi.org/10.14195/1647-8657_46_5

IÑAÑEZ, J. G., SPEAKMAN, R. J., BUXEDA I GARRIGÓS, J., GLASCOCK, M. D. 2009. "Chemical characterization of tin-lead glazed pottery from the Iberian Peninsula and the Canary Islands: initial steps toward a better understanding of Spanish colonial pottery in the Americas", Archaeometry, 51(4): 546-567. https://doi.org/10.1111/j.1475-4754.2008.00431.x.

LAFUENTE, P. 2010. "La producción cerámica sevillana durante la Baja Edad Media" consultado el 12 de mayo de 2019 en: http://www.retabloceramico.net/articuloo678.htm. https://doi. org/10.3989/sefarad.010.003

LANTES-SUÁREZ, O.; PRIETO-MARTÍNEZ, M. P.; MARTÍNEZ-CORTIZAS, A. 2011. "Aplicación de la Microscopía Electrónica de Barrido al estudio de los acabados de cerámica antigua de Galicia”, Gallaecia, 30: 117-125. https://doi.org/10.15304/gall.36.5137

LINARES, J.; HUERTAS, F.; CAPEL, J. 1983. "La arcilla como material cerámico. Características y comportamiento", Cuadernos de Prehistoria de la Universidad de Granada, 8: 479-490.

MARTÍNEZ CORTIZAS, A.; PRIETO LAMAS, B; LANTES SUÁREZ, O. Y PRIETO MARTÍNEZ, M. P. 2008. "Análisis elemental y cromático de cerámica prehistórica del área Ulla-Deza (NW P. Ibérica)”. En: S. Rovira Llorens, M. García Heras, M. Gener Moret e I. Montero Ruiz (eds.), Actas del VII Congreso Ibérico de Arqueometría, CSIC, Museo Arqueológico Nacional, Madrid, pp. 250264. https://doi.org/10.14195/1647-8657_46_5

MASON, R. B., TITE, M. S. 1997. "The beginnings of tin-opacification of pottery glazes", Archaeometry, 39(1): 41-58. https://doi.org/10.1111/j.1475-4754.1997.tboo789.x

MNCASGM. MUSEO NACIONAL DE CERÁMICA Y ARTES SUNTUARIAS GONZÁLEZ MARTÍ. (http://www.culturaydeporte.gob.es/mnceramica/colecciones/acceso-al-catalogo.htmly (consultado el 12 de mayo de 2019).

MOLERA, J., PRADELL, T., FARJAS, J., ROURA, P., SUREDA, M., ALBERCH, X., BURCH, J. 2005. "La cerámica dorada del Mas Llorens de Salt". En J. Molera, J. Farjas, P. Roura y T. Pradell (eds.), Avances en Arqueometría. Actas del VI Congreso Ibérico de Arqueometría. 16-19 Noviembre 2005, Universitat de Girona. Girona, pp. 109-120.

MOLERA, J., VENDRELL-SAZ, M., PÉREZ-ARANTEGUI, J. 2001. "Chemical and textural characterization of tin glazes in islamic ceramics from eastern Spain”, Journal of Archaeological Science, 28: 331-340. https://doi.org/10.1006/jasc.2000.0606. 
OLIVERA, C. O. 2000. El ocaso de las fortalezas compostelanas. Visitas y tasaciones (1535-1547). Cuadernos de Estudios Gallegos- Monografías 5. Santiago de Compostela.

Patrimonio Galego (http://patrimoniogalego.net/index.php/61727/2014/o6/castro-de-arreten-a-rocha-branca/) (consultado el 17 de marzo de 2016).

PÉREZ-ARANTEGUI, J., ORTEGA, J. M., ESCRICHE, C. 2005. "La tecnología de la cerámica Mudejar entre los siglos XIV y XVI: Las producciones esmaltadas de las zonas de Teruel y Zaragoza”. En J. Molera, J. Farjas, P. Roura y T. Pradell (eds.), Avances en Arqueometría. Actas del VI Congreso Ibérico de Arqueometría. 16-19 Noviembre 2005, Universitat de Girona. Girona,, pp. 89-96. https://doi.org/10.31819/9783964565297-013

POLVORINOS DEL RIO, A., CASTAING, J. 2010. "Lustre-decorated ceramics from a 15th to 16th century production in Sevilla”, Archaeometry, 52(1): 83-98. https://doi.org/10.1111/j.14754754.2009.00467.x

POLVORINOS, A., AUCOUTURIER, M., BOUQUILLON, A., CASTAING, J., CAMPS, J. 2011. "The evolution of lustre ceramics from Manises (Valencia, Spain) between the 14th and18th centuries", Archaeometry, 53(3): 490-509. https://doi.org/10.1111/j.1475-4754.2010.00552.x.

RICCI, C., BORGIA, I., BRUNETTI, B. G., SGAMELLOTTI, A., FABRI, B., BURLA, M. C., POLIDORI, G. 2005. "A study on late medieval transparent-glazed pottery and archaic majolica form Orvieto (Central Italy)”, Archaeometry, 47(3): 557-570. https://doi.org/10.1111/j.1475-4754.2005.00219.x.

ROMERO-PASTOR, J., GARCÍA-PORRAS, A., VAN GRIEKEN, R., POTGIETER-VERMAAK, S., COLL-CONESA, J., CARDELL, C. 2015. "New insgihts in thecnology characterization of medieval Valencia glazes", X-Ray spectrometry, 44(6): 426-435. https://doi.org/10.1002/xrs.2613.

SCHWEDT, A., MOMMSEN, H., ZACHARIAS, N., BUXEDA I GARRIGÓS, J. 2006. "Analcime Crystallization and compositional profiles-comparing approaches to detect post-depositional alterations in archaelogical pottery", Archaeometry, 48(2): 237-251. https://doi.org/10.1111/j.14754754.2006.00254.x.

TITE, M. S., FREESTONE, I., MASON, R., MOLERA, J., VENDRELL-SAZ, M., WOOD, N. 1998. "Lead glazes in antiquity -methods of production and reasons for use”, Archaeometry, 40(2): 241-260. https://doi.org/10.1111/j.1475-4754.1998.tboo836.x.

VILLA-AMIL Y CASTRO, J. 1907, Mobiliario Litúrgico de Galicia en la Edad Media, Madrid.

YZQUIERDO PERRÍN, R. 2006-2007. "El mecenazgo del arzobispo compostelano Don Lope de Mendoza en Santiago y Padrón”, Abrente: Boletín de la Real Academia de Bellas Artes: 38-39, 117-172. https://doi.org/10.21071/ucoarte.v3io.9494 